Volume 8. No. 5, May 2020

International Journal of Emerging Trends in Engineering Research

Available Online at http://www.warse.org/IJETER/static/pdf/file/ijeter49852020.pdf https://doi.org/10.30534/ijeter/2020/49852020

\title{
Steel Heat Treatment Technology
}

\author{
Smirnova Zhanna V. ${ }^{1}$, Vaganova O.I ${ }^{2}$, Kutepova L.I. ${ }^{3}$, Kuttepov M.M. ${ }^{4}$, Chaykina Zh.V. ${ }^{5}$ \\ ${ }^{1}$ Minin Nizhny Novgorod State Pedagogical University (Minin University), Nizhny Novgorod, Russian \\ Federation, z.v.smirnova@mininuniver.ru \\ ${ }^{2}$ Minin Nizhny Novgorod State Pedagogical University (Minin University), Nizhny Novgorod, Russian \\ Federation, vaganova_o@mail.ru \\ ${ }^{3}$ Minin Nizhny Novgorod State Pedagogical University (Minin University), Nizhny Novgorod, Russian \\ Federation, lubovkutepova@mail.ru \\ ${ }^{4}$ Minin Nizhny Novgorod State Pedagogical University, Nizhny Novgorod, Russian Federation \\ lubovkutepova@mail.ru \\ ${ }^{5}$ Minin Nizhny Novgorod State Pedagogical University, Nizhny Novgorod, Russian Federation \\ jannachaykina@mail.ru
}

\begin{abstract}
In the context of the development of the pace of modern production, the role of heat treatment is increasing, as the main method for producing steels with a given structure and mechanical properties. The parts and tools obtained because of heat treatment are widely used practically in all areas of human life. In Russia, up to $10 \%$ of melted steel grades are subjected to heat treatment hardening. For mechanical engineering, the volume of thermal redistribution exceeds $45 \%$ of the steel consumed by this industry. Both parts of devices, various parts of machines and large elements of equipment of metallurgical, transport, and energy industries undergo hardening. The main types of heat treatment are annealing, hardening, normalization and tempering, as well as thermomechanical processing of steel. In this paper, the main aspects of the theory and technology of heat treatment of steel are subject to consideration with a detailed consideration of the above methods of heat hardening of steels.
\end{abstract}

Key words: heat treatment, steel, metal properties, temperature.

\section{INTRODUCTION}

Heat treatment of steel allows giving products, parts and blanks the required qualities and characteristics. Depending on the stage at which the heat treatment was carried out in the manufacturing process, the workability of the workpieces is increased, residual stresses are removed from the parts, and the performance is improved for the parts.

Steel heat treatment technology is a combination of processes: heating, aging and cooling in order to change the internal structure of a metal or alloy. In this case, the chemical composition does not change.

The essence of heat treatment of steels is the change in grain size of the internal structure of steel. Strict adherence to temperature, time and speed at all stages, which directly depend on the amount of carbon, alloying elements and impurities that reduce the quality of the material. Structural changes occur during heating, which occur in the reverse order upon cooling.

\section{LITERATURE REVIEW}

Heat treatment of steel is carried out at temperatures close to critical points. This is where it happens:

-secondary crystallization of the alloy;

- transition of gamma iron to alpha iron state;

- transition of large particles into plates.

The internal structure of the two-phase mixture directly affects the performance and ease of processing.

The properties of metals and alloys depend not only on the chemical composition, but also on the structure. By heat treatment (heating to a certain temperature, holding at this temperature and selection

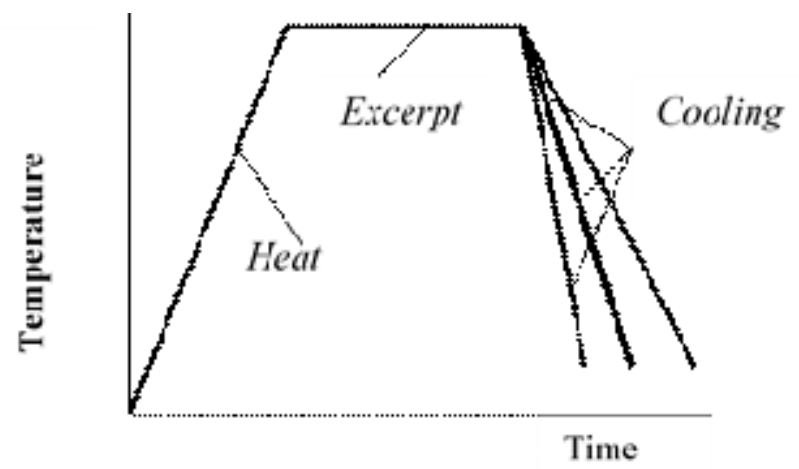

Figure 1: Scheme of heat treatment of metals and alloys

It can be seen from the Fe - C diagram (Fig. 1) that, as a result of slow cooling, hypereutectoid steels acquire the structure of ferrite and perlite, while hypereutectoid steels acquire the structure of perlite and secondary cementite.

Critical points are the temperature points at which these transformations occur:

Ac 1 , Ar 1 points on the PSK line;

$\mathrm{Ac} 2, \mathrm{Ar} 2$ points on the MO line; 
Ac3, Ar3 points on the GOS line;

Ac4, Ar4 points on the HJB line;

Acm points on the SE line.

Designations for heating lines - Ac and cooling - Ar are accepted. Above these points, the alloy will be in one phase, and below - in another. In this case, phase changes below the Ar lines occur in the solid state of the alloy.

There are several types of heat treatment (Fig. 2):

1. Recrystallization annealing (annealing of the first kind), phase transformations are absent in the alloys. It is used to reduce internal stresses, reduce hardness and increase ductility after cold working.

2. Annealing with phase recrystallization (annealing of the second kind). The heating temperature is higher than the temperature of phase transformations; therefore, phase changes occur. Cooling is slow. Get a fine-grained structure, relieve internal stresses.

3. Hardening. Heating above the phase transformation temperature and very fast cooling. As a result, phases and structures characteristic of high temperatures are fixed. Strength and hardness increase, but the structures are in a nonequilibrium state.

4. Vacation. Heating below the temperature of phase transformations in order to approach a stable equilibrium state. Plasticity increases, but hardness and strength decrease.

5. Chemical - heat treatment - saturation of alloys with carbon $\mathrm{C}$, nitrogen $\mathrm{N}$, sulfur $\mathrm{S}$ and other chemical elements by holding the alloys in gas, liquid or solid media [1].

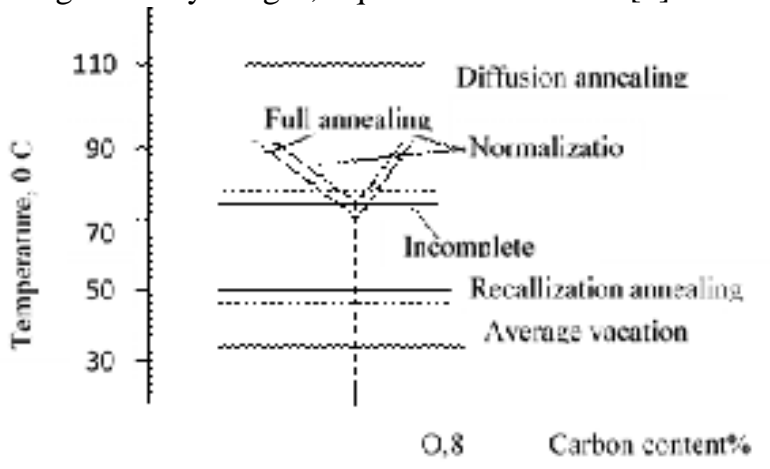

Figure 2: Steel heating temperatures for various types of heat treatment

Diffusion annealing of the first kind eliminates chemical heterogeneity in the ingots and castings; the metal is heated to a temperature Tn. of $80 \ldots 90 \%$ of the melting temperature Tm.

Recrystallization annealing eliminates hardening by heating the metal to a temperature $\mathrm{Tn}=\left(\begin{array}{lll}0.2 & \ldots & 0.6\end{array}\right) \mathrm{Tm}$. It is necessary for the preparation of blanks from rolled products for subsequent machining in the manufacture of parts [4].

Annealing is necessary to reduce residual stress in castings, welded structures, and after machining. It prevents warping and stabilizes the dimensions of the part.
Normalization is the heating of steel $30 \ldots 50^{\circ} \mathrm{C}$ higher than the critical points of Ac3 and Ac4, holding the required time and faster cooling in air than during annealing, therefore, finer-grained perlite is obtained, higher strength and hardness of the metal. The cooling of the part during annealing is carried out more slowly (the part is cooled together with the furnace) than during normalization (the part is cooled in air).

\section{RESULT AND DISCUSSION}

Tempered steel is subjected to tempering to increase its viscosity and ductility with a slight decrease in hardness and strength (Fig. 3). The part is heated to a temperature below Ac1 and cooled in air.

Low tempering $\left(150 \ldots 250^{\circ} \mathrm{C}\right)$ is carried out for products with high hardness (cutting tools: files, taps, dies). Average tempering $\left(350 \ldots 400{ }^{\circ} \mathrm{C}\right)$ is necessary for products with high elasticity and strength with sufficient viscosity (springs, springs,). High tempering $\left(450 \ldots 650{ }^{\circ} \mathrm{C}\right)$ gives the most viscous structure, but the smallest hardness and strength. (shafts, gears and other details). For alloy steels, the tempering temperature is selected higher than for carbon steels. Steel hardening.

Hardening and tempering are carried out in a complex in order to increase mechanical strength and hardness and maintain a sufficiently viscous structure [2].

Quenching is the heating of steel $30 \ldots 50{ }^{0} \mathrm{C}$ above the temperature of phase transformations, holding at this temperature and subsequent very quick cooling in water or oil. The structures obtained by rapid cooling are unstable; they represent various stages of austenite transformations (martensite, troostite, sorbitol).

The process of hardening steels has always been shrouded in mysticism, because man did not know the processes occurring in this. Recall the secret of damask steel [3].Damask steel was made before the new era, then the secret was lost, and only Russian metal expert Anosov P.P. in 1833 in the city of Zlatoust he discovered a secret. It turns out here a complex of effects on the metal: heating to a certain temperature, exposure (saturation of the surface of the blade with carbon in the forge) and stage-by-stage forging and hardening in several stages. The result is a viscous middle and high surface hardness of the blade of the blade due to saturation of the surface with carbon and hardening. 


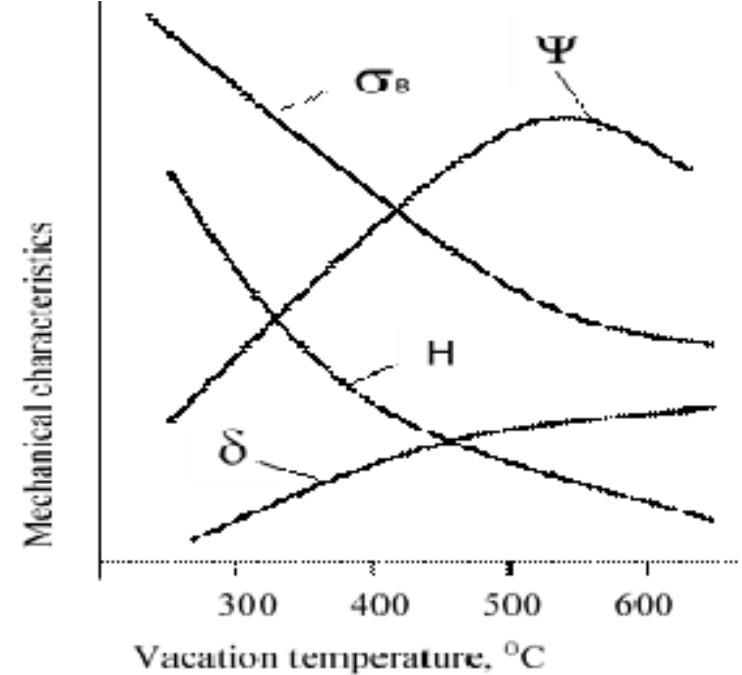

Figure 3: Effect of tempering temperature on the mechanical characteristics of steel

In the middle of the 17th century, "out of ignorance" they brought water to America from England in order to achieve the same quenching results as in England. In general, there was even an Arabic recipe for hardening of this kind: heat the dagger to incandescence, cool to the color of royal purple, immersing it in the body of a muscular slave. It was believed that the strength of the slave will turn into a dagger and give him hardness. There were also English patents recommending the addition of wildflowers to the cooling water [6].

The hypereutectoid steels (structural steels) during hardening are heated $30 \ldots 50^{\circ} \mathrm{C}$ above the Ac3 point; ferrite and perlite form a uniform austenite solid solution. If the metal is cooled slowly, then the usual phase transformations will occur in accordance with the iron-carbon diagram. With very rapid cooling, austenite does not have time to change its phase at temperatures below $727^{\circ} \mathrm{C}$, and in the temperature range of $300 \ldots 3500{ }^{\circ} \mathrm{C}$ and below, the so-called austenitic transformations occur and fine-needle martensite forms. It will be a complete hardening. And if you heat the steel in the range Ac1 ... Ac3, then there will be incomplete hardening (partial recrystallization of steel).

Eutectoid and hypereutectoid steels are also heated to a temperature $30 \ldots 500^{\circ} \mathrm{C}$ above Ac1, aged and quickly cooled. In hypereutectoid steel there will be: martensite, secondary cementite and residual austenite (incomplete hardening).

The part must be heated gradually and evenly so that there are no internal stresses, cracks and fractures. However, slow heating is a decrease in productivity, decarburization and surface oxidation.

The more carbon and alloying elements in steel, the more complex the form, the slower the part must be heated.

Protection against metal oxidation is carried out by using a protective gas environment $(\mathrm{CO} 2, \mathrm{~N}$,) or vacuum furnaces.

Cooling should have a high speed within $650 \ldots 5000 \mathrm{C}$ (so that there is no decomposition of austenite into a mixture of ferrite and cementite) and more slowly at $300 \ldots 200$ 0C, because In this area, with the formation of martensite, large internal stresses arise.

Water cools the part very well in the range of $650 \ldots 5500 \mathrm{C}$, but also too quickly in the range of $300 \ldots 2000 \mathrm{C}$, therefore mineral oils are used for hardening high-carbon and alloy steels.

Products of complex shape are quenched in two different liquid media or intermittently quenched (transfer to another cooling medium).

Self tempering. The "main" tools in construction so far are scrap, a chisel, a sledgehammer and a hammer, which should have high hardness on the surface and a relatively viscous core. They are not completely cooled during quenching, therefore, due to the heat of the inner layers of the part, metal is released (self-tempered).

Surface hardening. The metal surface is heated by high-frequency currents (including), gas burners and plasma. With surface hardening, warpage of the part is reduced and there is practically no scale. As a result, a viscous middle and a solid surface are formed. Moreover, the surface hardness will be higher than with conventional hardening.

Hardening followed by cold treatment is carried out for high-carbon steels, in which the temperature of the martensitic transformation of steels is in the negative temperature range.

During chemical - thermal treatment (CT) of steels, the chemical composition, structure, and properties of the surface layer change. As a result of HTO, the surface layer is hardened (hardness and strength, wear resistance increase) [7].

Types of CTO: cementation (saturation of the surface with carbon), nitriding (saturation with nitrogen), cyanidation (saturation with nitrogen and carbon), metallization, chromium plating, boronation, etc.

During cementation, the surface is carbonized to a depth of $0.5 \ldots 2 \mathrm{~mm}$ to increase the carbon content to $0.75 \ldots 1.2 \%$. Cementation is carried out in solid (charcoal), gas (natural gas, gas mixtures) and liquid media.

Nitriding is the saturation of steel or cast iron with nitrogen. Most often it is carried out in an atmosphere of ammonia NH3. The nitriding process is very long, so saturation with nitrogen to a depth of $0.5 \mathrm{~mm}$ must be carried out for at least 60 hours.

Cyanidation (solid, gaseous and liquid) of small and medium parts (gears, pistons, rings, rollers, etc.) is effective.

\section{CONCLUSION}

Thus, the hardenability of ordinary steels, even simple carbon ones, is higher than the required length of the hardened layer during surface hardening. Therefore, specially designed steels of low hardenability are used. Induction hardened steels are either carbon or low alloyed. At present, automatic lines 
Smirnova Zhanna V et al., International Journal of Emerging Trends in Engineering Research, 8(5), May 2020, 1790 - 1793

have been created and are operating, including all the necessary heat treatment processes.

\section{REFERENCES}

1.Kornilov Y.V., Ivanov I.A. Computer graphics training in additional education on the basis of inverted learning technology // Azimut of scientific research: pedagogy and psychology. 2019. Vol. 8.No. 4 (29). Pp. 111-114.

2. Smirnova Zh.V., Mukhina M.V., Katkova O.V., Gruzdeva M.L., Chernei O.T. Network interaction as a factor of professional qualities 'development of service workers // Lecture Notes in Networks and Systems (see books). 2020.Vol. 87.S. 698-704.

https://doi.org/10.1007/978-3-030-29586-8_81

3. A.A. Sokolovsky, A.I. Zemtsov, D.I. Kovalev, E.M. Shishkov, A.A. Kazantsev Hybrid Fiber Optic Temperature Sensor Powered by Optical Radiation // International Journal of Emerging Trends in Engineering Research Volume 7, No. 12 December 2019

https://doi.org/10.30534/ijeter/2019/217122019

4. Bartels, N. A. Metallography and heat treatment of metals / N.A. Bartels. - M.: State Scientific and Technical Publishing House, 2018. - 376 c.

5. Barkhatov N.A., Revunov S.E., Mukhina M.V., Gruzdeva M.L., Cherney O.T., Smirnova Z.V. Establishing the orientation of shock wave plane of solar wind magnetic cloud for conclusions about the level of auroral substorm activity JP Journal of Heat and Mass Transfer. 2019. Vol. 17. No. 1. S. 195-202.

https://doi.org/10.17654/HM017010195

6. Smirnova Zh.V., Cherney O.T. Technology of construction materials study guide / University of Minin. Nizhny Novgorod, 2019.

7. Y. Pratapa Reddy, Dr. K. L Narayana, Dr. M. Kedar Mallik.

Electro-chemical behavior of different metals in Sodium Chloride solution.// International Journal of Emerging Trends in Engineering Research, 7(11), November 2019, 71Volume 7, No. 11 November 2019. 